\title{
ANÁLISIS DE INVESTIGACIONES BRASILEÑAS ENFOCADAS EN EL CUIDADO DE ENFERMERIA, AÑOS 2001-2003\#
}

\author{
ANALYSIS OF BRAZILIAN NURSING CARE RESEARCH (2001-2003) \\ ALACCOQUE LORENZINI ERDMANN*,JOSETE LUZIA LEITE**, \\ ISABEL AMÉLIA COSTA MENDES***, MARIA AUXILIADORA TREVIZAN**** \\ y CLÁUDIA CARVALHO DANTAS*****
}

\begin{abstract}
RESUMEN
El cuidado de enfermería evolucionó en la búsqueda de nuevos saberes y quehaceres, en la perspectiva de reafirmar la profesión como ciencia, arte y tecnología. Este estudio es una investigación bibliográfica detipo descriptivo-exploratorio con abordaje cuanti-cualitativo que tiene como objetivos: Identificar las tesis de enfermería publicadas en los catálogos del Centro de Estudios e Investigaciones en Enfermería (CEPEn) en el período de 2001 a 2003, que abordaron el tema del cuidado; mostrar los referenciales teórico-metodológicos de dichas publicaciones; discutir las dimensiones del cuidado de enfermería surgidas de las tesis estudiadas. De los conocimientos nuevos obtenidos se encontraron 13 subunidades temáticas de las categorías asistencia einvestigación en enfermería. En estas temáticas convergen nuevos significados, concepciones, modos de pensar y hacer en la perspectiva de la construcción de nuevas tecnologías o saberes para la práctica deenfermería. Estos conocimientos encontrados presentan diferentes abordajes filosóficos y lineamientos metodológicos, nuevas técnicas y tecnologías en el campo teórico del saber cuidar, enfocando el cuidado en el quehacer dela enfermería en diferentes contextos institucionales y sociales.
\end{abstract}

Palabras claves: atención de enfermería; enfermería; investigación en enfermería.

\begin{abstract}
Nursing care has evolved in its search for new knowledge and practices, with a view to affirming the profession as science, art and technology. This study presents a descriptive and exploratory bibliographic research from a quantitative and qualitative approach. Objectives: to identify Brazilian nursing theses about care, disseminated in the catalogues of the Nursing Study and Research Centre (CEPEn) between 2001 and 2003; to disclose the theoretical and methodological referenceframeworks and therespectivenew knowledge produced in thesenursing theses; to discuss the new knowledge on care that emerged from these theses. Data were obtained from the

\#Investigación apoyada por el Consejo Nacional de Desarrollo Científico y Tecnológico (CN Pq).

* Enfermera. Profo. Titular dela Universidad Federal de Santa Catarina. Drª en Filosofía dela Enfermería. Coordinadora del Grupo de Estudios e Investigaciones en Administración de Enfermería y Salud (GEPADES). Coordinadora Adjuncta del Área de Enfermería de la CAPES Gestión 2001-2004 y Representante del Área Enfermería del CN Pq Gestión 2004-2007. Investigadora $1 \mathrm{~A}$ del CN Pq.

**Enfermera jubilada. Emérita de la Universidad de Rio de Janeiro (UNIRIO). M iembro de la Directoría del Núcleo de Investigación, Educación, Gerencia y Ejercicio Profesional de la Enfermería (NUPEGEPEn). Representante del Área de Enfermería del CN Pq, Gestión 1998-2001Investigadora 1A del CN Pq.

*** Enfermera. Profa Titular y Directora de la Escuela de Enfermería de Ribeirão Preto/Universidad de São Paulo (EERP/ USP) Centro Colaborador de la Organización Mundial de la Salud para el Desarrollo de la Investigación en Enfermería, Brasil. Representante del Área de Enfermería y Coordinadora del Comité Multidisciplinario de la Salud del CN Pq Gestión 2001-2004. Investigadora $1^{\text {A }}$ del CN Pq. Dirección para correspondencia: Av. Bandeirantes, 3900 - Campus Universitário Ribeirão Preto-SP - CEP 14040-902 - Brasil. Tel. 55-16-602-3393/Fax 55-16-633-3271. E-mail: iamendes@eerp.usp.br

**** Enfermera. Profa Titular y Líder del Grupo deEstudios el nvestigaciones sobrela Utilización de Recursos Humanos en Enfermería - Escuela de Enfermería de Ribeirão Preto/Universidad de São Paulo, Centro Colaborador de la OM S para el Desarrollo de la Investigación en Enfermería, Brasil. Investigadora 1A del CN Pq.

***** Enfermera. M aestran da del Curso de M aestría en Enfermería de la Escuela de Enfermería Anna Nery/ Universidad Federal de Rio de Janeiro (EEAN/UFRJ). Miembro del NU PEGEPEn. Beca CN Pq.
\end{abstract}


Brazilian Nursing Association's website, identifying 54 abstracts on care in a total of 301 theses. Different theoretical and philosophical referenceframeworks werefound, most of which adopted a socio-humanistic approach, favoring philosophers, anthropologists, sociologists, educators, historians and nurses. The new knowledge production gave rise to 13 thematic subunits of the categories nursing care and research: dialogical, humanistic, cross-dimensional, solidary care, self-care, care delivery/care process, care system, cultural care, care competencies, knowledge in care, power and care, care esthetics, care policies. These thematic subunits are an area of convergence for new meanings, conceptions and ways of thinking and doing with a view to constructing new technologies or knowledge for nursing practice. The knowledge found in these studies displays different philosophical approaches and methodological plans, new techniques and technologies in the theoretical field of care know-how and focuses on care in nursing practice in different institutional and social contexts.

Keywords: Nursing care, nursing, nursing research.

Fecha recepción: 30/11/04. Fecha aceptación: 19/08/05.

\section{CONSIDERACIONESINICIALES}

En pleno siglo XXI, entre conquistas como el mapeamiento de cromosomas que posibilitan la cura y tratamiento deenfermedades, los medicamentos de última generación para el combatea microorganismos multipotentes, la robótica que optimiza la realización de procedimientos quirúrgicos, se encuentra el cuidado, permeando por todos los pasos de esa evolución, sea en los actos, en las actitudes, en los momentos de atención, de dedicación y desvelo, representando la responsabilidad y acercamiento afectivo con el otro, ya sea directa o indirectamente (Boff, 1999).

Así, en medio detodos estos descubrimientos, seencuentra el cuidado. Secarece de nue vas formas de cuidar, nuevas visiones sobre los fenómenos tácitos a fin de mantenerse siempre acordes con los avances de las ciencias, de manera que acompañe la evolución de las nuevas técnicas y tecnologías emergentes, ya que el cuidar es la base de la profesión de enfermería (George, 1993).

El conocimiento teórico del cuidar empezó en enfermería en la década de los ' 50 con Madeleine Leininger, quien defendió la concepción de que el cuidado es una necesidad humana esencial, y el cuidar es la esencia de enfermería y es un modo de lograr salud, bienestar y la conservación de las culturas y de la civilización (Neves, 2002). Así como esta teó- rica, otrasenfermerastambién contribuyeron y han contribuido en la evolución de nuestra profesión. Sin embargo, se puede decir quela primera gran teórica, ejemplo y mito de la enfermería es Florence Nightingale, quien delineó los primeros conceptos de la profesión, reveló caminos de la investigación científica, enfatizando la observación sistemática como instrumento para la implementación del cuidado (Santiago \& Arruda, 2003).

Cabe resaltar que Florence no se preocupó en definir cuidar y cuidado, pero sí estableció los conceptos desalud y ambiente, surgiendo así la Teoría Ambientalista. Ella fue pionera en proponer y demostrar los efectos del ambiente del cuidado en la promoción de la salud delas personas, estableciéndosecomo marco en el inicio de la enfermería moderna.

Aunquela preocupación del carácter científico de la profesión se remonta a los tiempos de Florence Nightingale, destacamos que en Brasil la producción científica deenfermería es un fenómeno reciente que data de la década de los '50 (Angerami, 1993; Trevizan, Mendes y Angerami, 1991). Sin embargo, la enfermería brasileña inicia su práctica en el campo científico, legitimado por investigaciones de carácter científico permeadas por el método científico, en las décadas de 70 y 80 con la creación de los cursos de postgrado stricto sensu, maestría y doctorado (Santos, Souza y Oliveira, 2003). A partir de tales cur- 
sos se expandieron y profundizaron los conocimientos que fundamentan la profesión de enfermería, o sea, el cuidado de enfermería. Diversas enfermeras brasileñas han destacado esta temática en la orientación detesis de doctorado en el período de 2001-2003. Se puede decir que todas tienen espíritu de investigadoras, son verdaderas transformadoras sociales, responsables por la calidad del cuidado que se presta en el ámbito asistencial, en la enseñanza y/o la investigación, mediante la concretización y divulgación de sus estudios y reflexiones.

En virtud de su contexto histórico, la enfermería tiene su cuerpo de conocimientos relativamentenuevo y en plena construcción, el cual ha desarrollado a través de las investigaciones, especialmenteen las provenientes de loscursos dedoctorado. Cada trabajo producido avala la construcción y consolidación de su corpus teórico, añadiendo nuevas modalidades de cuidar, aportando mejorías para la calidad de la atención, enseñanza einvestigación con el consecuente mejoramiento de la profesión. Es importante mencionar que algunas investigadoras reconocidas señalan la necesidad de reunir proyectos con afinidades teóricas, a fin de profundizar estudios y avanzar en la construcción del conocimiento en enfermería(Gutierrez, Leitey Erdmann, 2002; M endes, 1991; M endes \& Trevizan, 1996).

A partir dela preocupación de conocer las investigaciones recientes de enfermería sobre el cuidado, estetrabajo busca investigar cuestiones con respecto a las dimensiones de los conocimientos producidos en las tesis brasileñas de doctorado, publicadas en los catálogos del Centro de Estudios e Investigaciones en Enfermería (CEPEn), en el período de 2001 a 2003, mostrando las referencias teóricas y metodológicas que permearon tales producciones.

En este sentido, el presente estudio tiene por objetivos: Identificar lastesis de enfermería publicadas en los catálogos del Centro de Estudios e Investigaciones en Enfermería (CEPEn), en el período desde2001 hasta 2003, que abordaron el tema del cuidado; mostrar los referenciales teórico-metodológicos de estas publicaciones; y discutir las dimensiones del cuidado de enfermería surgidas de las tesis estudiadas.

Considerar la naturaleza de cuidar (ontología) y su conocimiento (epistemología) como premisas que orientan y/o se presentan en otros estudios, hace relevantela realización deesta investigación, ya quepermite compartir tales aspectos. Estas premisas han sido blanco de interés y de investigaciones por abarcar aspectos éticos, estéticos, personales y empíricos (Silva \& Botoca, 2004), que hacen del cuidar un unificador e integrador de varios patrones del conocimiento (Waldow, 1998). En este contexto, la discusión acerca de esta temática se propone contribuir con la ciencia del "cuidar" y su consecutiva profundización teórica, destacándose por mejorar la práctica académica y profesional cotidianade la enfermería y demás ciencias afines.

\section{METODOLOGÍA}

Se trata de una investigación bibliográfica de tipo descriptivo-exploratorio, en la que se optó por un abordaje cuanti-cualitativo. El conjunto de datos cuantitativos y cualitativos se complementan de manera que abarquen dinámicamentela interacción entrelas informaciones recopiladas, excluyendo cualquier dicotomía existente entre los datos ( M inayo, 2000).

La fuente de datos de esta investigación la constituyeron los catálogos del Centro de Estudioselnvestigaciones en Enfermería (CEPEn) disponibles on-line, en el sitio de la Asociación Brasileña de Enfermería (ABEn) (Centro de EstudosePesquisasem Enfermagem, 2004). De esteacervo electrónico fueron recolectadaslas tesis que discutieran cuestiones relacionadas al cuidado deenfermería, correspondientes al periodo de 2001 a 2003.

Los datosfueron recolectados en el primer semestre de 2004, empleando un formulario 
con los siguientes ítemes: clasificación de la tesis en el catálogo, programa en lo cual fue desarrollada, orientador(es), título, referencial teórico y/o metodológico adoptado y el conocimiento que esta producción stricto sensu trajo para enfermería. Cabe señalar que esta recopilación fue procesada en dos fases distintas:

- Inicialmente, fueron leídos todos los resúmenes de las tesis (301 en total) contenidos en los catálogos on-line del CEPEn correspondientes a los años 2001 a 2003, separando aquellos que contenían como centro de la discusión el cuidado de enfermería.

- Los 54 resúmenes seleccionados que contenían la temática del cuidado fueron sometidosa repetidaslecturas, con la posterior discusión de cada resumen acerca del enfoque dado al cuidado.

- Tras la discusión detodos los resúmenes seleccionados, los principales aspectos fueron registrados en un formulario, cuyos ítemes (Anexo) fueron los especificados anteriormente.

Tras este primer momento, todos los formularios fueron sometidos nuevamente al proceso de lectura entre los autores del estudio, con la finalidad de optimizar el proceso de categorización y análisis estadístico. Dicho análisis fue realizado mediante cálculo porcentual, con base en las frecuencias absolutas, cuyo resultado está presentado en gráficos. En cuanto a la categorización, los conocimientos provenientes de las tesis analizadas fueron clasificados en las siguientes tres cate- gorías: enseñanza, investigación y asistencia.

Definidas de la siguiente manera:

- Enseñanza: Estudios sobreel cuidado en los que se resaltan experiencias inherentes a la enseñanza del cuidado, relacionada con cual quiera delos siguientes aspectos: formación derecursos humanos (nivel medio, pregrado, postgrado), currículo, asignaturas, estrategias de enseñanza, proceso enseñanzaaprendizaje, relación docente-alumno, aspectos individuales relacionados con el docente y/o el alumno.

- Investigación: Secomponedeestudios sobre el cuidado, enfocando diferentes abordajes filosóficos y lineamientos metodológicos, nuevas técnicas y tecnologías en el campo teórico del saber cuidar, encuestas y/o cuestionariossobrela investigación en enfermería.

- Asistencia: Estudios sobre el cuidado, sobre aspectos del quehacer de enfermería que involucre su actuación en el ámbito hospitalario, domiciliario, ambulatorio y comunitario.

\section{PRESENTACIÓN DE LOSRESULTADOS}

Fueron analizados 301 resúmenes de tesis de doctorado contenidos en los catálogos del CEPEn correspondientesalos años 2001, 2002 y 2003, entre los cuales se encontraron 54 resúmenes que tuvieron como tema central de la discusión el tema del cuidado. A continuación se presentan en figuras losresultados del análises de los 54 resumenes stricto-sensu brasileños (Gráfico I). 
Gráfico I. Distribución de tesis de enfermería brasileñas quetratan del tema del cuidado, publicadas en los catálogos on-line del CEPEn según las áreas de aplicación del conocimiento nuevo.

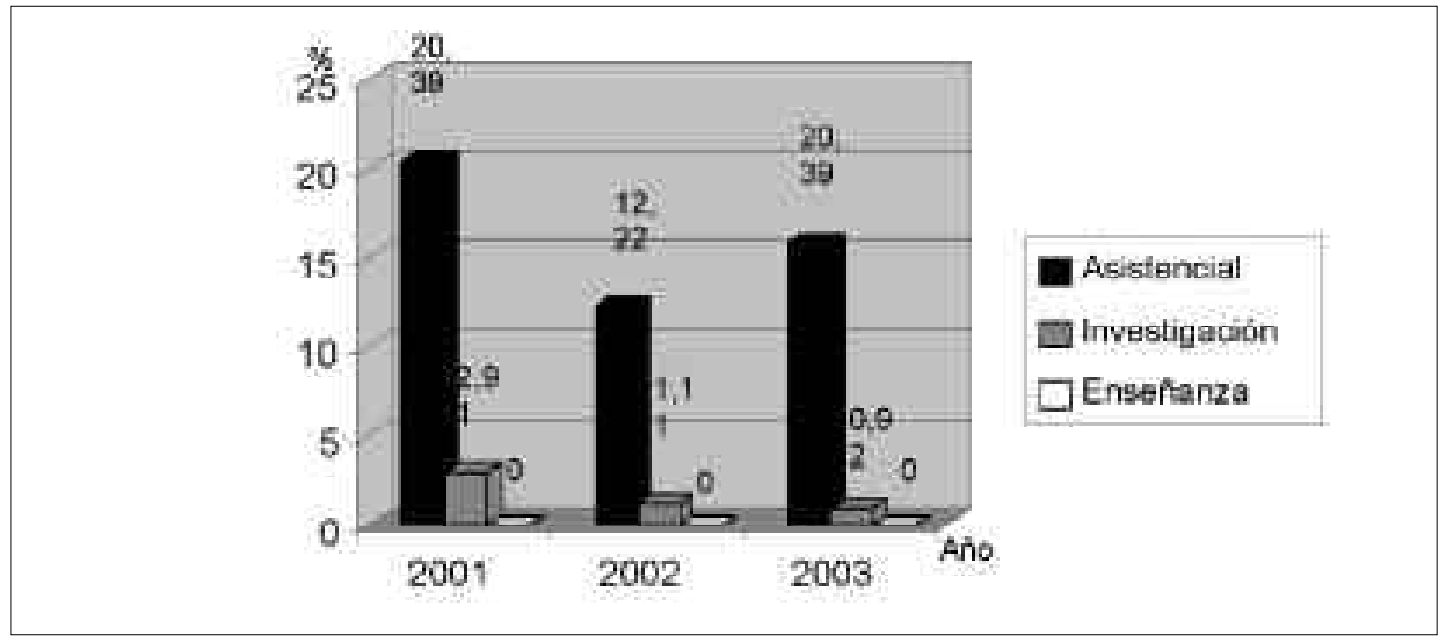

Fuente: Análisis de investigaciones brasileñas enfocadas en el cuidado de enfermería años 2001-2003.

El área de la asistencia de enfermería concentra casi la totalidad de las tesis publicadas en el período de 2001 a 2002; éstas tratan del cuidado relacionado con aspectos del quehacer de enfermería, involucrando su actuación en los ámbitos: hospitalario, domiciliario, ambulatorio y comunitario. La construcción deconocimientos sobreel quehacer o la práctica del cuidado muestra un aspecto impor- tante que caracteriza una real presencia de enfermería en estos escenarios. Del mismo modo, la insuficiente producción de conocimientos sobre la enseñanza del cuidado parece mostrar la dicotomía que todavía existe entre el hacer y el aprender o enseñar, o tal vez aún es muy fuerte la necesidad o prioridad de desarrollar más el hacer (Gráfico II).

Gráfico II. Distribución de la producción stricto sensu (tesis) brasileña que trata de la temática del cuidado publicada en los catálogos on-line del CEPEn según los programas de doctorado correspondientea los tres años analizados 2001-2003.

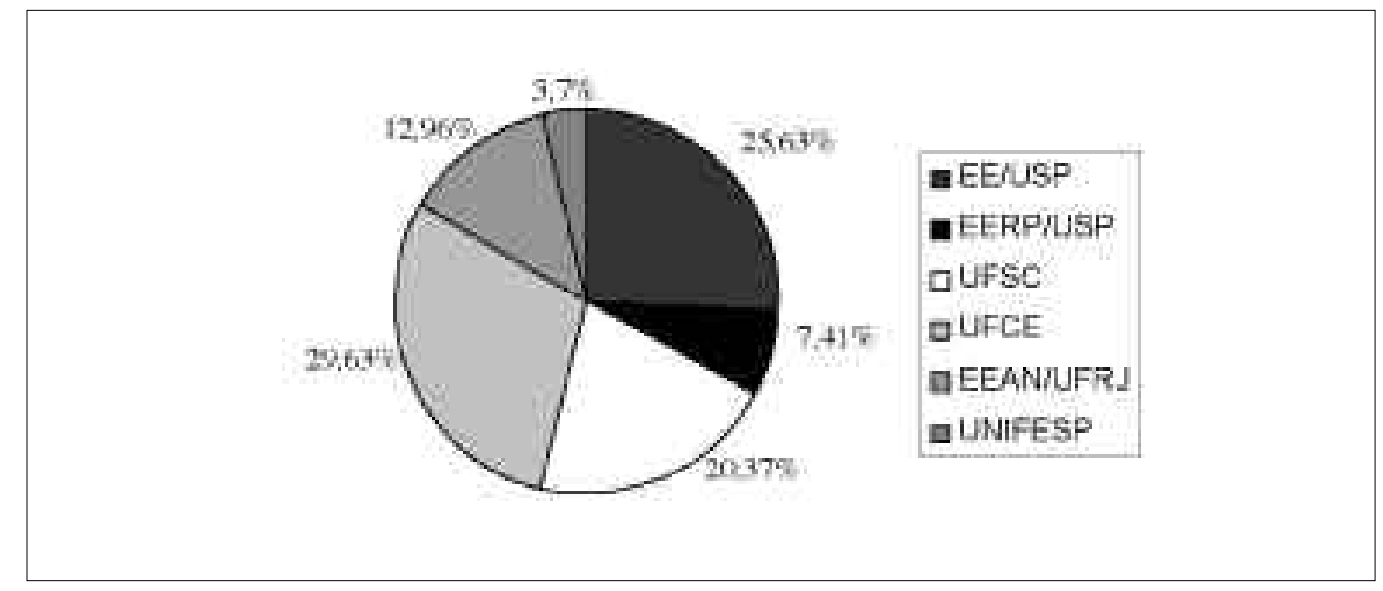

Fuente: Idem. gráfico I. 
Con respecto a los sitioso instituciones en los cuales estas tesis fueron hechas, se observó que el Programa de la UFCE presenta, en lostres años, un número mayor detesis sobre la temática del cuidado (29,63\%), seguido por la EE/USP con $25,63 \%$ y UFSC con $20,37 \%$.

Con relación a la distribución de las tesis por año de publicación, en el Gráfico 3 se observa quelos programas delaUFSC yUFC presentan un declive en investigaciones relacionadas con el cuidado, en tanto que el programa de la EE/USP se mantiene más armónico, con equilibrio en la cantidad de tesis a lo largo de los tres años analizados (Gráfico III).

Gráfico III. Distribución dela producción stricto sensu (tesis) brasileña quetratan dela temática del cuidado publicada en los catálogos on-line del CEPEn según los programas de doctorado.

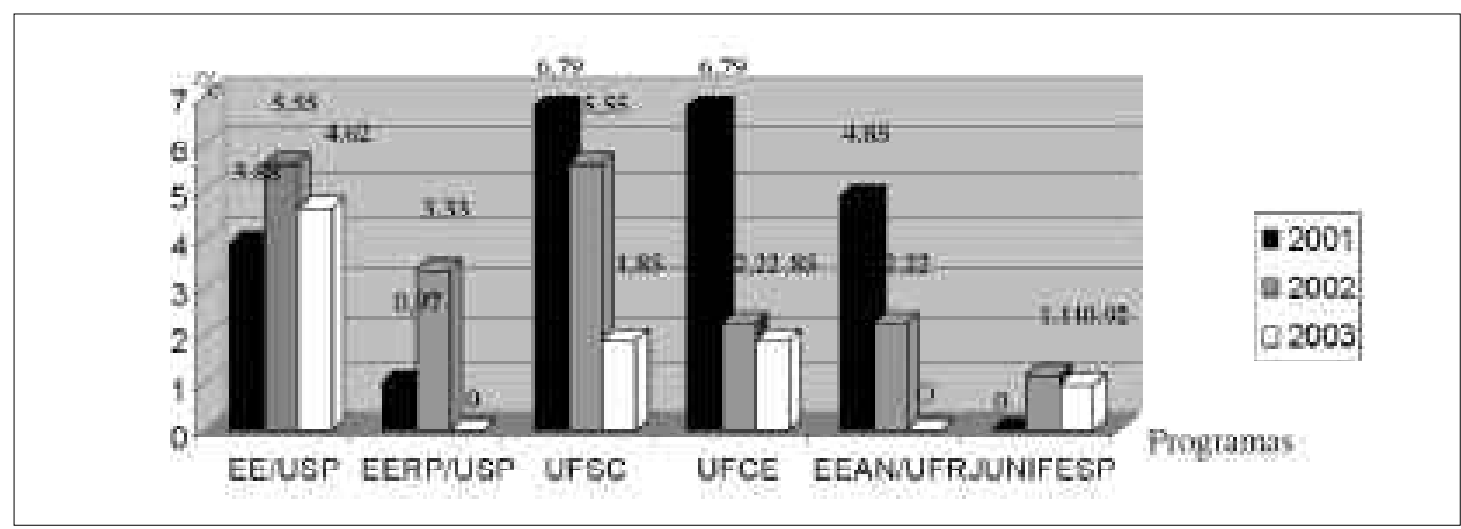

Fuente: Idem. gráfico I.

La distribución de la producción por re gión del país es mostrada en el Gráfico IV, en donde se observa que, no obstante que la mayoría de los Programas están situados en la región sureste, los dos de la región sur y el de la región noreste contribuyen a una distribución geográfica más homogénea, excluyéndoselas regiones nortey centro-oeste, queaún no posen Programas con Doctorado en Enfermería (Gráfico IV).

Gráfico IV. Distribución dela producción stricto sensu (tesis) brasileña quetrata dela temática del cuidado publicada en los catálogos on-line del CEPEn según año y regiones brasileñas.

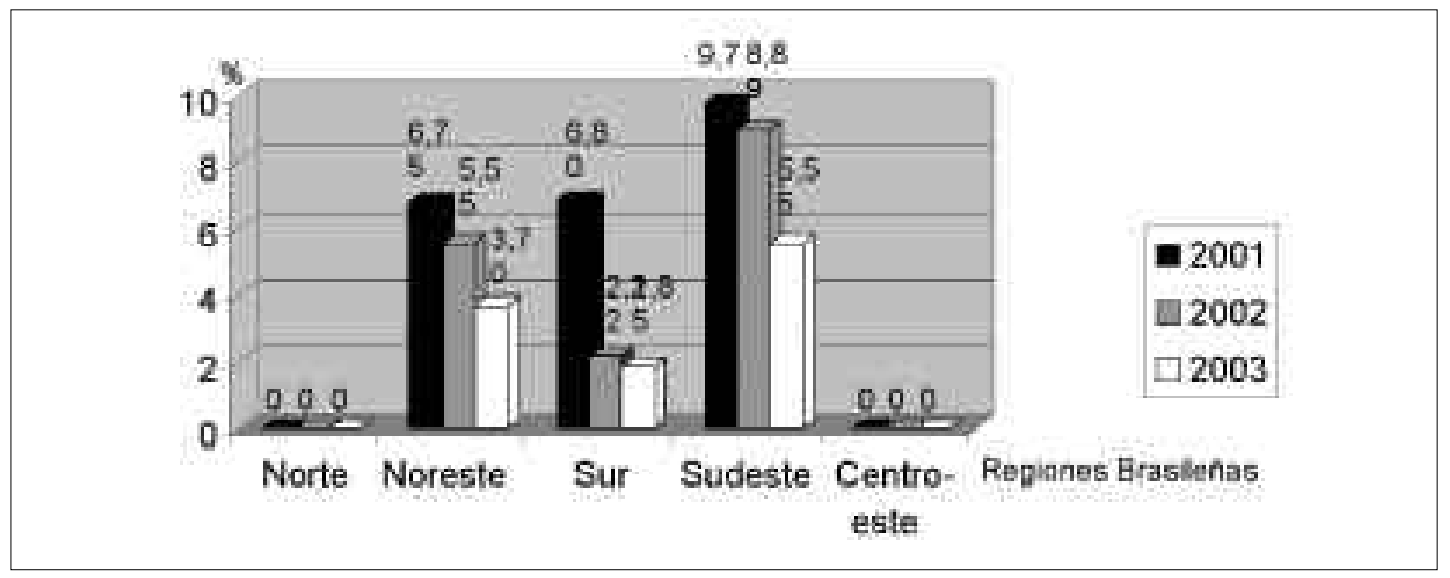

Fuente: Idem. gráfico I. 
Respecto a los referenciales teóricos y metodológicosutilizados, seencontró diversidad en los resúmenes, entre los que destacaron la Teoría Fundamentada en Datos (06 resúmenes), Interaccionismo Simbólico (05), Leninger (04), Paulo Freire (04), Orem (04), Dialéctica (03), Watson (02), Geertz (02), etnometodología (02), etnografía (02), representación social (02), historia oral (02) y Heidegger (02). Esta variedad de marcos referenciales indica una riqueza de bases que pueden ser utilizadas para construir conocimientos sobrela temática del cuidado. Los marcos referenciales teórico-filosóficos son de abordaje socio-humanista, privilegiando a los filósofos, antropólogos, sociólogos, educadores, historiadores y enfermeras.

En cuanto al objetivo de mostrar los resultados encontrados en las tesis en relación a los conocimientos producidos, son descritos abajo, agrupados por subunidades temáticas insertadas en las categorías de investigación y asistencia; están identificadas por números, de los cuales el primero se refiere al número de orden de registro de la recoplicación de datos, y el segundo al año en que fue publicada la tesis ( 2001 será 01, 2002 será 02 y 2003 será 03).

\section{Cuidado dialógico}

El diálogo en las relaciones de cuidado es evidenciado no sólo como estrategia comunicativa, sino también como un constructo que convergey concentra el propio cuidado, mostrándose como estructura y propiedad del cuidado, como contenido y forma, como procesos y contra-procesos de dialogicidad, implicando un pensar abierto, creativo y ético, ante de la autonomía y los derechos y deberes de los sujetos involucrados. Entre las tesis ubicadas en esta categoría, señalamos lo siguiente:
-El cuidado diálogo-libertador, que puede ser comprendido como aquel que involucra la sensibilidad, creatividad, dialogicidad y ética como características esenciales; y que es un acontecimiento capaz decontribuir a un nuevo modo depensar, sentir y actuar, en la perspectiva de promover el empoderamiento de la clientela. $(1,01)$.

\section{Cuidado humanístico}

El cuidado es realizado por seres humanos y el carácter humanístico marcó su historia, ahora es retomado no solamente para contraponer la tecnología de la máquina como materia física en la queel ser humano es cuerpo y materia, sino especialmente, para quelas acciones humanas no setornen mecanizadas. Las relaciones son atributos del ser en sus múltiples dimensiones devivir y convivir con otros, para sobrevivir y ser más saludable. Así, se destacan los siguientes resultados:

-Reconoce las dificultades y fallas en el proceso de cuidar. Sin embargo, proyecta una enfermería más humanizada a partir de la interacción entre enfermeras, con otros profesionales, pacientes y familiares. $(12,02)$. - La humanización dela atención necesita ser perfeccionada para ofrecer un cuidado integral a los niños hospitalizados. $(23,01)$.

\section{Cuidado transdimensional}

Las múltiples dimensiones del cuidado han permitido la comprensión de las dimensiones del ser humano y sus posibilidades de transcendencia, a partir desu unidad y totalidad.

\footnotetext{
-El cuidado transdimensional se traduce en un importantereferencial para el cuidado en psiquiatría. $(4,01)$.

- La forma de cuidar busca la unidad del ser a partir del evento dela enfermedad, como forma de promover la posibilidad de complementariedad entreel cuidar y curar. $(13,02)$.
} 


\section{Cuidado solidario}

En las relaciones de cuidado en que se muestra el cuidar de la vida y el sentido de la vida, permea el ser enfermero y su compromiso con ella. Una mirada ética a las condiciones humanas evidencia la importancia de la sensibilidad y de la conciencia solidaria para la construcción de paz de la civilización humana. El cuidado deenfermería setraduce como el cuidado con la vida, el cuidado con el ser humano.

-El modelo teórico demuestra conciencia solidaria en las relaciones del cuidado hospitalario, haciendo emerger el sentido dela vida. $(21,01)$.

- La revolución humana es la condición principal para desarrollar en el ser humano, la compasión queleimpulsa a cuidar de la vida, reconociendo en su vida y en la del otro una extrema dignidad y respeto. Esta revolución humana partedeuna persona, seamplíapara toda la sociedad y para todo el cosmo, proveendo así la paz mundial. $(7,03)$.

\section{El autocuidado}

En el proceso de vida del humano, las relaciones de dependencia son necesarias para la dinámica de la vida, en el proceso de nacer, crecer y morir, incluso para trascender. Esta dependencia implica la competencia de autonomía del ser en relación al otro, mediante el intercambio, la ayuda, la colaboración y la construcción de nuevas posibilidades de ser, con competencias para cuidar de sí, no de pendiendo del otro, pero con la contribución del otro para fortalecer sus competencias para cuidar. La necesidad decuidar de otro está en la dependencia de las personas para la atención a sus necesidades básicas, mientras que el cuidado de sí posibilita la autonomía del ser, estar y sobrevivir. Deestemodo, el cuidado es representado por actitudes, preocupación, responsabilidad y compromiso con el otro y, al mismo tiempo, responsabilidad consigo mismo.
-El proceso de acción educativa participativa vislumbra una forma renovada de práctica de enfermería, que contempla la especificidad del ser humano anciano, práctica participativa, dialogada y construida con los sujetos del auto-cuidado, el anciano hospital izado y su familiar acompañante. $(3,01)$.

- Los paraplégicos sometidos a un Programa Educativo quetiene por objetivo el auto-cuidado relacionado con los déficits identificadosen la eliminación intestinal, consigueprogresos en queasuma el auto-cuidado. $(12,03)$.

\section{El proceso de cuidar/cuidado}

El cuidar y el cuidado se constituyen en el hacer de la enfermería como un modo, una forma, una manera, un estilo, un patrón, una dimensión, un sistema, una estructura, un proceso, u otros, con actitudes y estrategias formaleseinformales, visibles, sentidas, percibidas o no, que configura la práctica del ser-haciendo a través de la salud de los sereshumanos en su proceso devivir. La vida, entendida como el proceso de vivir se sustenta sobre el continuo proceso de cuidado mutuo y simultáneo desí, de los otros y por los otros, de las formas vivas (seres vivos) y de los seres inanimados (mundo material físico) (Erdmann, 1996).

- Modelo teórico: teniendo que cuidar - la vivencia del anciano y de la familia cuidadora en el proceso decuidar y ser cuidado en contexto domiciliario. $(18,01)$.

- La cotidianeidad de la familia del niño que vivencia una situación de dolor como consecuencia de artritis reumatoide juvenil, es estructurada en torno de dos elementos (motivacionales eintervenientes) y una categoría central, el cuidado, para que el niño crezca a pesar del dolor, quesetransformó en un componente esencial delo cotidiano de la familia que tiene bajo su cuidado un niño con artritis reumatoide juvenil. $(6,03)$.

\section{Sistema de cuidado}

El cuidado ocurre en losseres, a partir deellos y a través de ellos, coexistiendo en la natura- 
leza donde sus estructuras pueden ser pensadas, ya que están presentes en la organización dela vida de los seres, en sus dominios biológicos, antropológicos, psicológicos, sociológicos y otros. Así, la concepción de un sistema de cuidado es posible a partir del rastreo desu red simultánea deinter-retroacciones de sus movimientos ondulantes en el establecimiento de límites internos y externos, cuyos sentidos y significados resultan de propiedades todavía desconocidas por la ciencia, incluyendo sus posibles procesos auto-eco-organizadores. Es posible visualizar un sistema de cuidados de enfermería que se conforma por movimientos ondulantes de relaciones, interacciones y asociaciones en estructuras y propiedades de procesos auto-eco-organizadores de dimensiones variadas de cuidado. Estas abarcan desdeel cuidar de sí, desí junto con el otro, de sentir el sistema personal de procesar el cuidado del cuerpo por sí mismo, deser y estar en el sistema de relaciones múltiples del cuidado, hasta la dimensión del cuidado con la naturaleza, integrándose con los demás sistemas sociales y naturales, fortaleciendo el sentido de pertenencia y acercando a los seres en la búsqueda de mayor sobrevivencia, vida y civilización humana (Erdmann, 1996).

- La red de saberes de las madres es formada con la experiencia empírica y la vivencia. (9, $01)$.

-El padecer de las familias rurales se deriva de elementos inhibidores como la falta de comunicación e información entre el ambiente y el trabajo. El descuido en relación a la salud surge en consecuencia de la fragilidad de las interrelaciones de los sistemas. (5, 02).

\section{El cuidado cultural}

Los conocimientos sobre la antropología de la salud apuntan hacia la importancia del cuidado congruente entre las creencias y valo- res, hábitosy costumbres de los diferentes grupos sociales. La diversidad cultural en nuestro país, sea a través dela influencia traída por losinmigranteso a través delos pueblos nativos, constituye una riqueza de prácticas en salud, importantes y necesarias para el conocimiento científico, acercando creencias y saberes a los quehaceres más particulares y adecuados a las necesidades de los grupos poblacionales.

\begin{abstract}
-En la práctica de educación en salud, los profesionales no consideran la diversidad de condiciones socioculturales que involucran al niño en su contexto familiar; siguen los protocolos institucionales y no integram el saber popular al saber científico, aprovechando como fundamento de valor las experiencias de las familias. $(11,03)$.

- La transformación del cuidado constituye la formación social del ostomizado, cuando son val orados losfactores culturales se amplia su seguridad y confort. $(16,02)$.
\end{abstract}

\section{Competencias para el cuidado}

La competencia profesional para el cuidado demanda un ejercicio crítico, reflexivo y constructivo sobre las prácticas disciplinares e interdisciplinares en salud, siendo capaz de conocer e intervenir en el proceso de vivir, enfermar y estar sano, con compromiso y responsabilidad en la búsqueda del mejoramiento de la vida. El trabajo de enfermería se centra en el cuidado, en las dimensiones de cuidar, gerenciar, educar e investigar, sustentado por los diversos patrones de conocimientos, competencias y habilidades específicas (M endes, Trevizan y Lourenço, 2002; Trevizan \& Mendes, 1993; Trevizan \& M endes, 1995).

- La búsqueda del ejercicio del cuidar como expresión de un saber cualificado. $(14,01)$. - Necesidad de transformación en la acción del profesional de enfermería en el sentido de mejorar el cuidar y cuidado del parapléjico hospitalizado. $(11,02)$. 


\section{El conocimiento en el cuidado}

Aunquela práctica del cuidado sea milenaria, los fundamentos teóricos y filosóficos sobre la misma aún están en fase de construcción. Las tecnologías del cuidado aún son incipientes y requieren un avance en la sustentación teórica. Las teorías de enfermería ya consolidan sus disciplinas propias, pero todavía carecen de estudios que propicien su aplicabilidad en nuestra realidad (Mendes, Leite, Trevizan, Trezza y Santos, 2002). Los siguientes resultados contribuyen al avance del conocimiento sobre el cuidado.

- La construcción de los patrones de calidad para queel cuidado deenfermería pueda contribuir a la prevención y control de infecciones en cirugías ortopédicas y promover un cuidado más libre de riesgos, los cuales re presentan el todo del proceso del cuidado en esta Unidad de trabajo en salud. $(1,03)$. - La aplicación dela Acajumembrana en clientes con heridas crónicas favoreció la eliminación del dolor, ardor, sensación de quemazón, exsudado, prurito, edema, regeneración gradual de los tejidos destruidos por el proceso inflamatorio y cicatrización de la herida, lo que finalmente favoreció a una mejor calidad de vida de los clientes de esta investigación. (15, 02).

\section{El poder y el cuidado}

Las relaciones de poder en el cuidado son importantes para una práctica más socializada y de mayor consenso entre los sujetos involucrados. El ejercicio del cuidado se identifica como un ejercicio de poder, de dependencia o de autonomía, mediado por relacionesfacilitadoras o dificultoras de este proceso, sustentadas por dominios de conocimientos, dominios de fuerzas articuladas, o creencias y prácticas habituales, entreotros aspectos, los cuales carecen de mayor conocimiento para una convivencia más civilizada entre los seres humanos.
- Las relaciones entre las madres y el equipo de enfermería se muestran complejas y permeadas por el ejercicio del poder, siendo necesario buscar la construcción compartida. $(13,01)$.

-M oviéndose entre la libertad y la reclusión: viviendo una experiencia de pocos placeres para quien es familiar cuidador de una persona con AVC. $(16,01)$.

\section{La estética del cuidado}

El cuidado es sentido. Las sensaciones y percepciones en el cuidado posibilitan el sentir, el vivir, la naturaleza humana, la naturaleza de la vida, las diversas formas o modos de vivir, el placer deser y estar percibiendo y el sentido de vivir, el existencial del ser y el cuidado como una posibilidad deser, dellegar a ser y deconstruir nuevos modos de vivir. El imaginario social, lasimágenes sobresí y sobreel mundo dan sentido al cuidado y a la vida.

- La estética delosolores: el sentido do olfato en el cuidado de enfermería hospitalaria. $(20,01)$. -El horizonte del cuidado se debe localizar antela posibilidad de transcender los límites establecidos como posibilidad, pensar en la reconstrucción, en la cual las necesidades humanas de salud de las personas deben orientar el camino, en el que el arte, ocupando su lugar, va a crear un espacio de convergencia con la ciencia, lo que permitirá el surgimiento del arte de cuidar y el cuidado humano. $(4,03)$.

\section{Políticas de cuidados}

Las articulaciones propiciadas en las relaciones del cuidado conforman su carácter político, posibilitando su presencia en las políticas o pensamientos que orientan la propuesta de las prácticas sociales, incluyendo las prácticas de salud de los ciudadanos. El sujeto determinado y determinante pueden contribuir, o sea, participar en las decisiones para el vivir individual y colectivo, con dignidad y 
respeto. Los resultados delas tesis de enfermería muestran al gunas posibilidades de avance en estos aspectos.

- Valoración de la concordancia del cuidado deenfermería con las expectativas delas personas, con la finalidad de no ser destituido de la condición de ser humano. $(17,02)$. - Construcción de un modelo teórico, que muestre la lucha constante de la enfermera recién formada para reestablecerse con la cohesión y flexibilidad posibles, poniendo en práctica el cuidado. Sin embargo, ampliando y redimensionando más allá del cuidado ideal con la perspectiva de aumentar competencias, ya sean clínicas o administrativas. $(9,02)$.

\section{CONSIDERACIONES FINALES}

A través de las tesis de enfermería, el presente estudio evidenció que la construcción de conocimientos acerca del cuidado en Brasil se concentra en el área asistencial. En el total de los tres años investigados, el Programa de la UFCE presenta un número mayor de tesis sobre la temática cuidado $(29,63 \%)$, seguido de la EE/USP y UFSC con $25,63 \%$ y $20,37 \%$, respectivamente.

En las 54 tesis de doctorado encontradas, deun total de 301 resúmenes existentes en los catálogos del Centro de Estudios e Investigaciones en Enfermería (CEPEn) disponibles on-line, se identificó una diversidad de referenciales teóricos y metodológicosutilizados, lo que indica una variedad de bases que pueden ser utilizadas para construir conocimientos sobre la temática del cuidado.

Delas dimensiones de conocimientos producidos, surgieron 13 subunidades temáticas de las categorías de asistencia einvestigación, las cuales muestran una diversidad de aspectos o dimensiones del cuidado en dichas categorías, como producto de los nuevos conocimientos generados en losúltimos tres años.

De acuerdo con la caracterización de las categorías de este estudio, los conocimientos encontrados presentan diferentes abordajes filosóficos y lineamientos metodológicos, nuevas técnicas y tecnologías en el campo teórico del saber cuidar. Tales conocimientosfue ron generados a partir de estudios sobre el cuidado enfocado a diferentes aspectos del hacer relacionado a la actuación de la enfermería en losámbitos hospitalario, domiciliario, ambulatorio y comunitario.

Las perspectivas de avance en estas temáticas son prometedoras, ya que en ellas convergen nuevos significados, concepciones y modos de pensar y hacer posible la construcción de nuevas tecnologías o saberes para la práctica de la enfermería.

Esteestudio pretendió mostrar los referenciales teórico, metodológicos y los conocimientos producidos en las tesis de enfermería deBrasil, publicadas en el período de 2001 a 2003, que abordaron la temática del cuidado como una primera búsqueda de informaciones. Sin embargo, no serealizó una lectura completa de las tesis, y en los resúmenes podrían estar omitidas o incompletas informaciones importantes.

\section{REFERENCIAS BIBLIOGRÁFICAS}

Alligood, M. R., Chong-Choi, E. (1994). Evolución del desarrollo de las teorías de enfermería. In A. M arriner-Tomey. Modelosy teorías en enfermería. (3 ed). Madrid. M osby/D oyma Libros.

Angerami, E. L. S. (1993). O desenvolvimento da pesquisa no Brasil. Revista Latino-americana de Enfermagem 1, (especial), 43-52.

Arendt, H. (1997). A condição humana. Rio de Janeiro RJ. Forense Universitária.

Boff, L. (1999). Saber Cuidar: Ética do humano compaixão pela terra. Petrópolis RJ. Editora Vozes.

Capra, F. (1996). A teia da vida: uma nova compreensão dos sistemas vivos. São Paulo SP. Cultrix.

Centro de Estudos e Pesquisas em Enfermagem (CEPEn). (2004). Catálogos. Recuperado em 2004 de http://www.abennacional.org.br

Collièrre, M . F. (1999). Promover aVida: da prática das mulheres de virtude aos cuidados de enfermagem. ( 3 ed). Lisboa. LIDEL.

Erdmann, A. L. (1996). Sistema de cuidados de enfermagem. Pelotas. Universitária/U FPel.

Freitas, K. S. S. (2000). O cuidado no processo de ser e viver de educandas de enfermagem Disser- 
tação não publicada. UFSC, Florianópolis SC.

George, J. B. (1993). Teorias de Enfermagem: Os Fundamentos à Prática Profissional. Porto Alegre RS. Artes M édicas.

Guerrero, A. Z. (2003). El cuidar de si como valor em enfermería Tese não publicada. Universidad de Carabobo, Venezuela.

Gutiérrez, M. G. R., Leite, J. L., Erdmann, A. L. (2002). Os múltiplos problemas pesquisados ea pesquisa em enfermagem. Revista Brasileira de Enfermagem 55,(5), 535-541.

Mendes, I. A. C. (1991). Pesquisa em enfermagem: impacto na prática. São Paulo SP. EDUSP.

Mendes, I. A. C. (1994). Enfoque humanístico à comunicação em enfermagem. São Paulo SP. Sarvier.

Mendes, I. A. C. (2000a). 0 talento humano ao encontro da qualidade eda ética do cuidado. Revista Latino-americana de Enfermagem 8, (6), 1-5.

Mendes, I. A. C. (2000b). O resgate do cuidar na enfermagem. Revista Latino-americana de Enfermagem 8, (3), 1-5.

Mendes, I. A. C., Trevizan, M. A. (1996). The evolution of nursing research in Brazil. In J. J. Fitzpatrick \& J. Norbeck. Annual Review of Nursing Research (pp. 225-242). N ew York. Springer Publishing.

Mendes, I. A. C., Trevizan, M. A., Nogueira, M. S., Sawada, N . O. (1999). Humanizing nurse-patient communication: a challengeand a commitment. Medicine and Law 18, (1), 639-644.

Mendes, I. A. C., Trevizan, M. A., Lourenço, M. R. (2002). A gerência esclarecida no trabalho do enfermeiro. Revista Latino-americana de Enfermagem 10, (5), 704-708.

Mendes, I. A. C., Leite, J. L., Trevizan, M . A., Trezza, M. C. S. F., Santos, R. M . (2002). A produção tecnológica e a interface com a Enfermagem. Revista Brasileira de Enfermagem 55, (5), 556-561.

M inayo, M. C. S. (2000). Pesquisa social: teoria método e criatividade. (15 ed). Petrópolis RJ. Vozes.

Neves, E. P. (2002). As dimensões do cuidar em enfermagem: concepções teórico-filosóficas. Escola Anna Nery Revista de Enfermagem 6; (suplemento 1), 79-92.
Reich, W. T. (1995). History of notion of care. In Bioethics encyclopedia. (2 ed). New York. M ac Millan Library.

Santiago, M. M. de A., Arruda, A. A. (2003). Interdisciplinaridade na produção do conhecimento deenfermagem. EscolaAnna Nery Revista de Enfermagem 7, (2), 167-175.

Santos, Z. M ., Sousa, A., Oliveira, V. L. M. de. (2003). A pesquisa de enfermagem e 0 impacto do conhecimento produzido. EscolaAnna Nery Revista de Enfermagem 7, (2), 176-185.

Silva, D. M., Botoca, E. M.V. (2004). 0 conhecimento científico ea enfermagem. Recuperado em 2004, de http://www.ipv.pt/millenium/M illenium27/ default.htm.

Trevizan, M. A., Mendes, I. A. C. (1993). Administration of patient care: theoretical aspects. International Nursing Review 40, (1), 25-28.

Trevizan, M. A., M endes, I. A. C. (1995). Perspectives of participative management in nursing. Applied Nursing Research 8, (4), 156-159.

Trevizan, M . A., M endes, I. A. C., Angerami, E. L. S. (1991). A investigação em enfermagem no Brasil. Revista Paulista de Enfermagem 10, (3), 9195.

Universidad de la Sabana . Faculdad de Enfermería. (2003). ¿Qué Significa Aquichan? Revista Aquichan 3, (3). Recuperado em outubro 2004 de http://www.unisabana.edu.co/aquichan/ aquichan que.htm

Villalobos, M. M. D. (1998). Enfermería: desarrollo teórico e investigativo. Bogotá. Editorial Unibiblos/Universidad Nacional de Colombia.

Waldow, R. (1992). Cuidado: uma revisão teórica. Revista Gaúcha de Enfermagem 13, (2), 29-35.

Waldow, R. (1998). Cuidado humano. 0 resgate necessário. Porto Alegre. Sagra-Luzzatto.

Waldow, R. (1998). Examinando o conhecimento na enfermagem. In D. M eyer, R. Waldow, M. J. Lopes. $M$ arcas da diversidade: saberes e fazeres da enfermagem contemporânea. Porto Alegre RS. Artmed.

Zoboli, E. L. C. P. (2003). A redescoberta da ética do cuidado: o foco e a ênfase nas relações. Revista da Escola de Enfermagem da U SP 38, (1), 21-27. 